\title{
VIP activates primordial follicles of rat through ERK-mTOR pathway in tissue culture
}

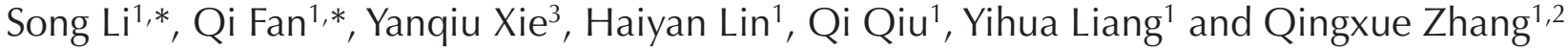 \\ ${ }^{1}$ Department of Obstetrics and Gynaecology, IVF Centre, Sun Yat-Sen Memorial Hospital, Sun Yat-Sen University, \\ Guangzhou, China, ${ }^{2}$ Guangdong Provincial Key Laboratory of Malignant Tumour Epigenetics and Gene Regulation, \\ Sun Yat-Sen Memorial Hospital, Sun Yat-Sen University, Guangzhou, China and ${ }^{3}$ Department of Obstetrics and \\ Gynaecology, IVF Centre, Guangdong General Hospital, Guangzhou, China
}

Correspondence should be addressed to Qingxue Zhang; Email: zhangqingxue666@aliyun.com

*(S Li and Q Fan contributed equally to this work)

\begin{abstract}
In vitro activation of primordial follicles is becoming more essential in assisted reproductive technologies. Vasoactive intestinal peptide (VIP) is one of the members of the neurotrophin family which has demonstrated to have an impact on follicle development in recent years. This study aims to investigate the effect of VIP on the activation of primordial follicles in neonatal rat in an in vitro culture system and to determine the relevant molecular mechanism of their activation. Ovaries of 4-day-old rats were examined for the expression of VIP receptors and were cultured in mediums containing VIP with or without inhibitors of the ERK-mTOR signalling pathway. They were then collected for histological analysis or measurement of the molecular expression of this pathway. The receptors of VIP were found in granular cells and oocytes of primordial and early-growing follicles in neonatal ovary. The ratio of growing follicle increased in the presence VIP at different concentrations, with the highest level of increase being observed in the $\mathbf{1 0}^{-7} \mathrm{~mol} / \mathrm{L}$ VIP-treated group. The ratio of PCNA-positive granular cells was also increased, while that of the apoptotic oocytes were decreased, and protein analysis showed increased phosphorylation of ERK1/2, mTOR and RPS6 in the VIP-treated group. However, the effect of VIP on the activation of primordial follicle became insignificant with the addition of MEK inhibitor (U0126) or mTORC1 inhibitor (rapamycin). This study indicated that VIP could activate neonatal rat primordial follicle through the ERK-mTOR signalling pathway, suggesting a strategy for in vitro primordial follicle recruitment.

Reproduction (2019) 157 475-484
\end{abstract}

\section{Introduction}

Cryopreservation of ovarian tissue is an important way to preserve fertility in women who are facing fertilitythreatening diseases or treatments (Ladanyi et al. 2017, Kim et al. 2018). However, re-transplantation of ovarian tissue may carry the risk of malignancy relapse. Poor surgical procedure or limited ovarian tissue might also cause infertility after re-transplantation (Kim et al. 2018). To solve this problem, researchers are progressively exploring suitable ways to obtain matured oocytes through in vitro activation (IVA) of primordial follicle. Some researchers have obtained ovary tissue from patients with ovarian insufficiency for IVA that involves Hippo signalling disruption and protein kinase B (AKT) stimulation, followed by auto-transplantation and assisted reproductive technologies. Even though live birth was achieved after re-transplantation of the cultured ovarian tissue, the low success rate made it necessary for new IVA strategy to be developed (Suzuki et al. 2015, Zhai et al. 2016). In view of the complexity of primordial follicle activation, the application of inhibitor or stimulator of a key signalling pathway may not be sufficient. According to literature, a series of growth factors are involved in follicle development (Zhang \& Liu 2015), but how these factors influence the activation of primordial follicles remains unclear. Therefore, revealing the functions and mechanism of these factors may be of great benefit to primordial follicle development.

Vasoactive intestinal peptide (VIP), which is mainly produced by the central and peripheral nervous system as a neuroendocrine hormone, a neurotransmitter or a cytokine, has many important biological effects including relaxing the blood vessels, promoting gastrointestinal peristalsis, regulating the immune system and protecting the nervous system (Onoue et al. 2008). In recent years, VIP was found to be associated with the ovary function (Streiter et al. 2016). The mRNA transcripts or protein of VIP and its receptors, VPAC1 and VPAC2, were identified in the ovaries of some mammals (Hulshof et al. 1994, Barberi et al. 2007, Gabbay-Benziv et al. 2012, 
Bukowski \& Wąowicz 2015). Many studies have since showed that VIP might influence the development of preantral and antral follicles (George \& Ojeda 1987, Mayerhofer et al. 1997, Cecconi et al. 2004). We have previously demonstrated that VIP could promote the development of neonatal rat ovary during in vitro culture (Chen et al. 2013). However, the expression and localization of proteins for VIP and its receptors in neonatal rat ovary, and the exact mechanism by which VIP influences the recruitment of primordial follicle has not yet been revealed.

VIP phosphorylates various proteins mainly through the cyclic adenosine monophosphate (cAMP)-protein kinase A (PKA) signalling pathway after binding to VPAC and produces physiological effects, such as vasodilation and immune regulation, promoting digestion (Couvineau \& Laburthe 2012). Mitogen-activated protein kinase (MAPK), one of the downstream proteins of PKA, has been implicated as a key regulator of cell proliferation and differentiation (Pearson et al. 2001). One of the three major classes of MAPKs in mammals is mitogen-activated protein kinases 3 and 1(MAPK3/1) (also known as extracellular signal-regulated kinases 1 and 2 (ERK1/2)), of which phosphorylation is mediated by a MAPK kinase (MAPKK, otherwise known as MAPK-ERK kinase 1, MEK1) (Davis 2000). Previous studies have shown that ERK1/2 took part in the activation of primordial follicles (Zhao et al. 2018). Zhang et al. recently found that the recruitment of primordial follicles is triggered by mTORC1 activation in pregranular cells in a geneknockout rat model (Zhang et al. 2014). A study has suggested that ERK1/2 could regulate the activity of mTORC1 in the activation of primordial follicles (Zhao et al. 2018); therefore, we hypothesize that VIP could promote the recruitment of primordial follicles through the ERK-mTORC1 signalling pathway.

The activation of primordial follicles is the first step in follicle development to produce a viable oocyte. This highlights the importance of elucidating the regulatory mechanism involved in order to provide theoretical support for preserving female fertility and treatment of infertility. In this study, we examined the expression of VIP and its receptors in the neonatal rat ovary and revealed the mechanism of how VIP affected the development of primordial follicle.

\section{Materials and methods}

\section{Animals and reagents}

Four-day-old Rattus norvegicus (Sprague-Dawley) female rats were obtained from the Centre of Experimental Animals, Sun Yat-Sen University. They were housed under controlled conditions of temperature $\left(20-26^{\circ} \mathrm{C}\right)$, relative humidity $(35-$ $75 \%)$ and photoperiod (12L: 12D), with free access to food and tap water and housed with their mother. Experimental protocols were approved by the Institutional Animal Care and Use Committee (IACUC) of Sun Yat-Sen University.

The reagents used in the present study were obtained from the following sources and handled as follows: vasoactive intestinal peptide (VIP) (1911) from Tocris Bioscience (Bristol, UK) was dissolved to $3.0067 \times 10^{-4} \mathrm{~mol} / \mathrm{L}$ in water; $\mathrm{VIP}_{6-28}(\mathrm{~V} 4508)$ from Sigma-Aldrich was dissolved to $1.775 \times 10^{-3} \mathrm{~mol} / \mathrm{L}$ in water; U0126 (\#9903) from Cell Signaling Technology was dissolved to $0.01 \mathrm{~mol} / \mathrm{L}$ in DMSO; Rapamycin (53123-88-9) from Gene Operation (Michigan, USA) was soluble in DMSO at $0.055 \mathrm{~mol} / \mathrm{L}$. All reagents mentioned above were stored under $-20^{\circ} \mathrm{C}$ conditions and diluted to suitable concentration in the culture medium (see below).

\section{Ovary culture}

We had previously successfully established an in vitro ovary culture system (Chen et al. 2013). Ovaries were dissected from 4-day-old female rats using small dissecting instruments and isolated in phosphate-buffered saline (PBS, Gibco BRL) under stereomicroscope (SteREO Discovery V.12, ZEISS). The ovaries were then washed three times with warmed sterile PBS. Whole ovaries were randomly distributed into groups and two ovaries were placed in a Millicell-CM filter insert each (pore size, $0.4 \mu \mathrm{m}, 3413$, Corning) floating on the culture medium in individual wells of a 24-well culture dish (Corning), which was previously equilibrated for $30 \mathrm{~min}$ with $600 \mu \mathrm{L}$ of culture medium, ensuring that the ovaries were completely covered by medium. The plates were incubated at $37^{\circ} \mathrm{C}$ with $5 \% \mathrm{CO}_{2}$. The medium consisted of a mixture of Dulbecco's modified Eagle medium plus F-12 medium (1:1 v:v; Gibco BRL) containing $0.05 \%$ bovine serum albumin (Sigma-Aldrich), $1 \%$ ITS-X (effective concentrations: $10 \mathrm{mg} / \mathrm{mL}$ of insulin, $5.5 \mathrm{mg} / \mathrm{mL}$ of transferrin, and $6.7 \mathrm{ng} / \mathrm{mL}$ of sodium selenite; Gibco BRL), $0.05 \mathrm{mg} / \mathrm{mL}$ of L-ascorbic acid (Sigma-Aldrich), antibiotics $(100 \mathrm{U} / \mathrm{mL}$ of penicillin, $100 \mu \mathrm{g} / \mathrm{mL}$ of streptomycin and $5 \mu \mathrm{g} /$ $\mathrm{mL}$ of Fungizone (amphotericin B), all from Gibco BRL). The culture medium was replaced with fresh medium daily. After a 3-day in vitro culture, ovaries were immediately placed into $4 \%$ paraformaldehyde (PFA, Leagene, Beijing, China) for histological follicle assessment or stored in $-80^{\circ} \mathrm{C}$ for western blotting or qPCR.

\section{Ovarian histomorphology}

The ovaries were fixed in $4 \%$ PFA for $24 \mathrm{~h}$, and then dehydrated, paraffin-embedded and cut into $5 \mu \mathrm{m}$ sections. The sections were rehydrated and stained with hematoxylin and eosin $(\mathrm{HE}$, Leagene, Beijing, China) for morphological observation and differential follicle counts. Follicle counting was performed on every fifth section of the ovaries by two independent investigators who were blinded to the experiment. Follicle stages were identified according to the following definitions: the primordial follicle is counted when the nucleus is surrounded by a flat layer of pre-granular cells; primary follicle is an oocyte encircled by a single layer of cuboidal granular cells; secondary follicle has at least two layers of cuboidal granulosa cells without antrum and the antral follicle has an antrum. Both primary follicles and secondary follicles are 
grouped as early-growing follicles. Occasionally, follicles may consist of both cuboidal and flat squamous granulosa cells, which indicate an intermediate stage between the primordial and primary stages. In these cases, stage identification was decided according to the type of predominant granular cells (Chen et al. 2013).

\section{Immunohistochemistry and immunofluorescence}

After deparaffinization and rehydration, the sections were incubated in citrate buffer $(\mathrm{pH}$ 6.0, Wuhan Servicebio Technology Co., Ltd., China) and were microwaved for $10 \mathrm{~min}$, and then immersed in $3 \% \mathrm{H}_{2} \mathrm{O}_{2}$ for 10 min at room temperature to block endogenous peroxidase activity. Nonspecific binding was blocked with $10 \%$ normal goat serum (Beijing Biosynthesis Biotechnology Co., Ltd. (Bioss), Beijing, China) for $1 \mathrm{~h}$ at $37^{\circ} \mathrm{C}$. The sections were incubated with the primary antibody (antiVIP (1:200, ab8556), anti-VPAC1 (1:200, ab245743), antiVPAC2 (1:200, ab216630) and anti-PCNA (1:400, ab92552)) (Abcam) for $24 \mathrm{~h}$ at $4{ }^{\circ} \mathrm{C}$ followed by room temperature placement for $30 \mathrm{~min}$. For immunohistochemistry, horseradish peroxidase (HRP)-combined secondary antibodies (Zhongshan Golden Bridge Bio-technology, Beijing, China) were added for a 30 -min reaction at $37^{\circ} \mathrm{C}$, followed by diaminobenzidine (DAB, Zhongshan Golden Bridge Bio-technology) staining. The sections were counterstained with hematoxylin and then dehydrated and mounted with neutral balsam. For immunofluorescence, Cy3-conjugated secondary antibodies (Jackson ImmunoResearch Laboratories, Inc.) were added at $37^{\circ} \mathrm{C}$ for $1 \mathrm{~h}$, followed by 4,6-diamidino-2-phenyiindole (DAPI, BestBio, Shanghai, China) staining for $5 \mathrm{~min}$. The sections were washed three times ( 5 min each) with PBS after each of the above procedure. At the same time, a negative control was treated by replacing primary antibodies with PBS. Finally, the sections were observed and photographed under the microscope.

\section{TUNEL assay}

In Situ Cell Death Detection Kit-POD (Roche), a commercial kit of TUNEL assay, was employed to evaluate cell apoptosis of follicles. After dewaxing and rehydrating, the sections were incubated in citrate buffer ( $\mathrm{pH}$ 6.0) and heated by microwave for $10 \mathrm{~min}$, and then rinsed twice with PBS. A $50 \mu \mathrm{L}$ TUNEL working solution per sample was mixed with label solution and enzyme solution, while the negative control used label solution. The sections were incubated at $37^{\circ} \mathrm{C}$ for $60 \mathrm{~min}$ in the dark, followed by rinsing twice with PBS. Then the nuclei were counterstained with DAPI for $10 \mathrm{~min}$ and washed three times with PBS (5 min each). Finally, the fluorescence of the sections was photographed and analysed by fluorescence microscope (ZEISS).

\section{Western blotting}

Ovaries were placed in RIPA lysis buffer (Beyotime Institute of Biotechnology, Shanghai, China) with protease inhibitor PMSF (Beyotime Institute of Biotechnology) and pulverized by ultrasonic wave on ice, followed by centrifugation at $13,400 \mathrm{~g}$ and $4^{\circ} \mathrm{C}$. After that, protein content in the liquid supernatant was determined using a BCA Protein Assay Kit (ComWin Biotech Co., Ltd, Beijing, China) and $30 \mu \mathrm{g}$ of protein from each sample were loaded onto $6-10 \%$ SDS-PAGE (Ten ovaries were used in each sample.). The proteins of interest were separated by electrophoresis, and transferred to PVDF $(0.22 \mu \mathrm{m})$ membranes. The membranes were then blocked in $5 \%$ bovine serum albumin solution (Gibco BRL, dissolved in Tris-buffered saline containing $0.1 \%$ Tween 20 ) for $1 \mathrm{~h}$ and probed with specific primary antibodies overnight at $4{ }^{\circ} \mathrm{C}$. Primary antibodies against ERK1/2(1:1000, 4695, CST, Boston, MA, USA), phosphor- ERK1/2 (Thr202/Tyr204) (1:1000, 4370, CST), mTOR (1:2000, 2972, CST), phosphor-mTOR (Ser2481) (1:1000, 2974, CST), RPS6(1:2000, 2217, CST), phosphorRPS6 (Ser235/236) (1:2000, 2211,CST), AKT (1:1000, 4691, CST), phosphor-AKT (Ser473) $(1: 1000,2211$, CST), Forkhead box O3 (FOXO3A) (1:1000, 12829, CST), phosphor-FOXO3A (Ser253) (1:1000, ab47285, Abcam), PCNA (1:1000, ab152112, Abcam) and $\beta$-actin $(1: 10,000,1970$, CST) are all rabbit antibodies. HRP-conjugated goat anti-rabbit IgG (1:5000, 133997, Jackson ImmunoResearch Laboratories Inc.) was used to determine the proteins and an ECL kit (Millipore) was employed for visualization. ImageJ (National Institutes of Health, Java image processing software) was used to quantify the integrated light intensity of each band and to determine the concentration of phosphoproteins as well as the changes induced by treatment and calculate the ratio of phosphorylated proteins to their non-phosphorylated forms. $\beta$-actin expression was used as an internal control.

\section{Real-time quantitative RT-PCR (qPCR)}

First, total RNA was isolated from ovaries using RNA prep pure Micro Kit (Qiagen) in accordance with the manufacturer's protocols. RNA concentration was then measured with NANODROP 2000 (Thermo Scientific). Next, the extracted RNA (100 ng each for $20 \mu \mathrm{L}$ reaction) was reverse-transcribed into cDNA using Transcriptor First Strand cDNA Synthesis Kit (Roche) according to the manufacturer's instructions. Finally, the reverse transcription products were used as a template for PCR amplification with SYBR Premix Ex Taq II (TaKaRa). The following primer sequences were used: GAPDH: forward 5'-GGATGGAATTGTGAGGGAGA-3' and reverse 5'-GTGGACCTCATGGCCTACAT-3'; Sohlh 1: forward 5'-GAGAGAACGCAGGAGGAGGA-3' and reverse 5'-GACATCTCCAGGACGGAAGC-3'; Amh: forward 5'-TGGCTGAAGTGATATGGGAGC-3' and reverse 5'-TAGCACCAAATAGCGGGTGTC-3'. Each reaction well in a 96-well unclear plate (Axygen, San Francisco, CA, USA) contained $0.4 \mathrm{pmol} / \mu \mathrm{L}$ forward and reverse primers, $1 \times$ HotstartFluo-PCR mix (TaKaRa) and $10 \mathrm{ng}$ cDNA. The final reaction volume was $20 \mu \mathrm{L}$ and all samples were run in triplicates. The amplification of cDNA was performed under the following conditions: pre-denaturation at $95^{\circ} \mathrm{C}$ for $30 \mathrm{~s}$, followed by 40 cycles of denaturation at $95^{\circ} \mathrm{C}$ for $3 \mathrm{~s}$ and annealing at $60^{\circ} \mathrm{C}$ for $30 \mathrm{~s}$ in LightCycler 480 II (Roche). Data were collected and used for relative quantitative analysis with the $2^{-\Delta \Delta C T}$ method. Relative mRNA expression level was obtained by comparing data of the experimental group with 
those of the control group and gene expression was normalized to Gapdh. The experiment was done in triplicate.

\section{Statistical analysis}

All experiments were carried out in triplicates. Statistical analyses were performed using IBM SPSS Statistics for Windows, version 22.0 (IBM Corp.). Data were expressed as means \pm S.D. and analysed by one-way ANOVA or nonparametric equivalent tests. Turkey test was used to compare two groups within three or four treatments. $P<0.05$ was considered statistically significant.

\section{Results}

\section{The expression of VIP and its receptor in the 4-day-old rat ovary}

VIP takes effect mainly via its receptors 1 and 2 (VPAC1 and VPAC2), so we first examined whether these two receptors exist in the neonatal rat ovary by using antiVPAC1 or VPAC2 antibody. IHC results showed that VPAC1 was mainly expressed in the cell membrane of granular cells and oocytes both in primordial and earlygrowing follicles, without positive stain in the ovarian cell nuclei or stroma. The expression pattern of VPAC2 was similar to that of VPAC1. There was no positive stain for VIP (Fig. 1). The specificity of the VPAC antibodies was confirmed by Western blot (Supplementary Fig. 1, see section on supplementary data given at the end of this article).

\section{The effect of VIP on the activation of primordial follicle in neonatal rat ovary}

To explore the effects of VIP on follicle development and to determine the suitable concentration for an in vitro ovarian culture system, we cultured the 4-day-old ovaries for 3 days with different concentrations of VIP $\left(10^{-6} \mathrm{~mol} / \mathrm{L}, 10^{-7} \mathrm{~mol} / \mathrm{L}\right.$ and $\left.10^{-8} \mathrm{~mol} / \mathrm{L}\right)$. Hematoxylin and eosin (H\&E) analysis showed that there were more early-growing follicles and less primordial follicles in the VIP-treated ovaries (Fig. 2A and B). The ratios of early-growing follicle/primordial follicle (GF/PF) in VIPtreated groups were higher compared with those of the control group (base culture medium without VIP), especially for the $10^{-7} \mathrm{~mol} / \mathrm{L}$ VIP-treated group (Fig. 2C). To prove the specificity of VIP, ovaries were pre-treated with $5 \times 10^{-6} \mathrm{~mol} / \mathrm{L} \mathrm{VIP}{ }_{6-28}$, the specific antagonist of VIP receptors, for $2 \mathrm{~h}$, and then co-treated with VIP and $\mathrm{VIP}_{6-28}$ for 3 days. In the VIP $+\mathrm{VIP}_{6-28}$ group, HE analysis showed less early-growing follicles as compared with the VIP-treated group (Fig. $3 \mathrm{~A}$ and $\mathrm{B}$ ); the ratio of GF/PF was similar to the control groups, which dropped to nearly half of that in the VIP-treated group (Fig. 3C). This outcome indicates that the activation of primordial follicle by VIP could be inhibited by $\mathrm{VIP}_{6-28}$ and that VIP acted specifically through its receptors VPAC1 and VPAC2 in the ovary.

As the activation of primordial follicles includes granular cell proliferation and oocyte growth (Zhang \& Liu 2015), we then examined the effect of VIP on granular cell proliferation through proliferating cell nuclear antigen (PCNA) analysis and examined oocyte apoptosis using TUNEL assay. Compared to the control group or $\mathrm{VIP}+\mathrm{VIP}_{6-28}$ group, more primordial follicles were observed to be PCNA-positive per section in the VIP group $(53.2 \% \pm 5.9 \%$ vs $32.0 \% \pm 4.0 \%, 36.9 \pm 2.9 \%$, respectively). Result from Western blot also showed that VIP treatment increased the expression of PCNA by 1.5fold compared to that of the other two groups (Fig. 4A, $\mathrm{B}$ and $\mathrm{C}$ ). The ratio of apoptotic oocytes per section was $20.4 \pm 3.5 \%$ in the VIP group, which is lower than the rest $(49.2 \pm 4.1 \%, 38.5 \pm 2.6 \%)$ (Fig. $4 \mathrm{D}$ and $\mathrm{E}$ ).

Spermatogenesis and oogenesis-specific basic helixloop-helix 1 (Soh/h1), a germ cell-specific gene that regulates the activation of primordial follicles, is mainly expressed in germ cell cysts and oocytes of primordial follicles in the ovary (Pangas et al. 2006, Jagarlamudi \& Rajkovic 2012), while anti-Mullerian hormone (Amh) (Dayal et al. 2014), the key mediator of early follicular differentiation, is expressed in granular cells of growing follicles. We thus examined the mRNA expression of

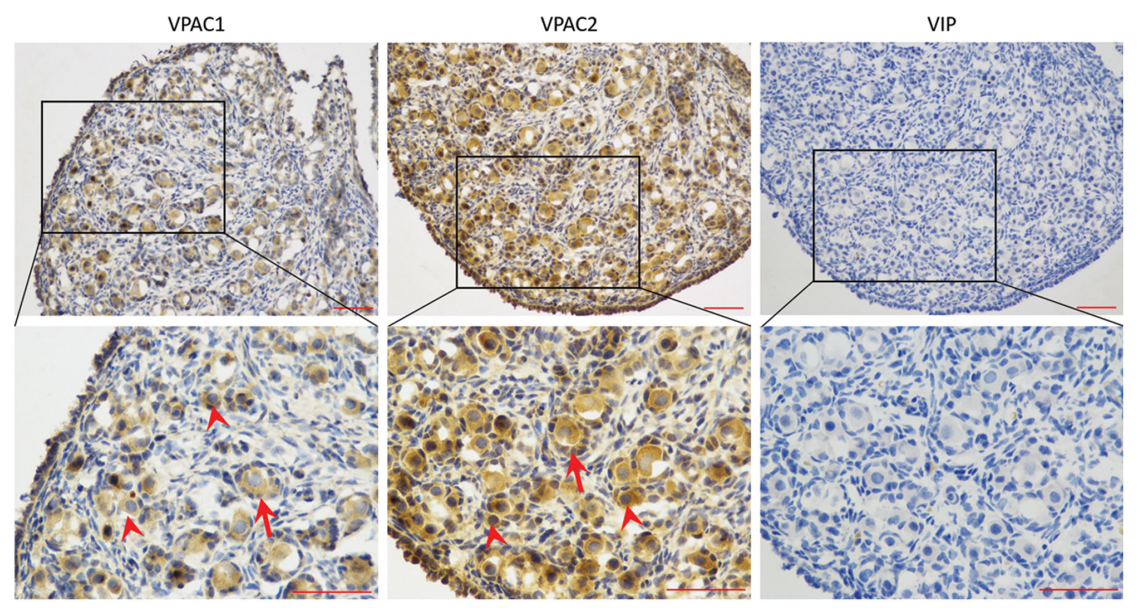

Figure 1 The expression of vasoactive intestinal peptide (VIP) and its receptors in 4-day-old rat ovary. Four-day-old rat ovaries were freshly collected and cut into $5 \mu \mathrm{m}$ sections, followed by immunohistochemistry for detecting VIP and its receptors - VPAC1 and VPAC2 (arrowheads, positive primordial follicle; arrows, positive early growing follicles). Scale bar: $50 \mu \mathrm{m}$. 
A
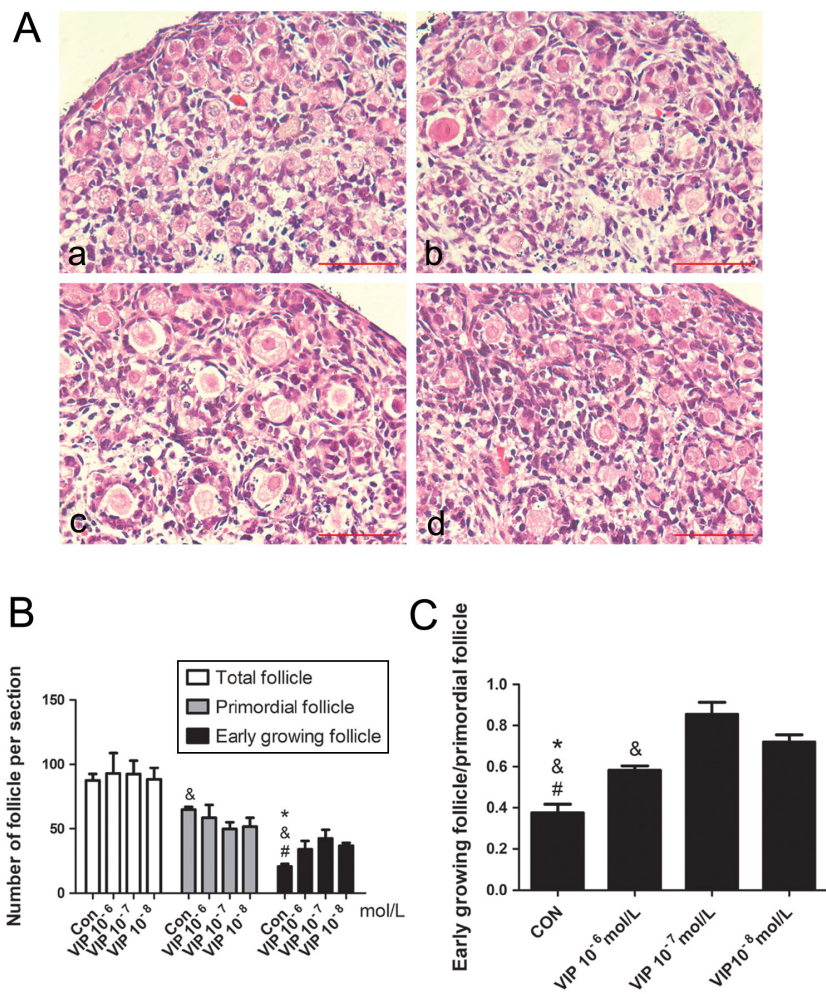

Figure 2 VIP promoted the transformation from primordial follicles to early growing follicles in neonatal rat ovary. Four-day-old rat ovaries were cultured in basal medium without VIP (control group) or with VIP in different concentrations for three days. (A) The representative photos of all groups stained with hematoxylin and eosin: control (a), $10^{-6} \mathrm{~mol} / \mathrm{L}(\mathrm{b}), 10^{-7} \mathrm{~mol} / \mathrm{L}(\mathrm{c}), 10^{-8} \mathrm{~mol} / \mathrm{L}(\mathrm{d})$. (B) and (C) The numbers of early-growing follicles and primordial follicles were counted, and their ratio was calculated. Scale bar: $50 \mu \mathrm{m}$. Values are mean \pm S.D. of at least three experiments. $\left({ }^{*} P<0.05\right.$ compared with the $\mathrm{VIP}^{-6} \mathrm{~mol} / \mathrm{L}$ group, ${ }^{\&} P<0.05$ compared with the $\mathrm{VIP}^{-7} \mathrm{~mol} / \mathrm{L}$ group, ${ }^{\sharp} P<0.05$ compared with the $\mathrm{VIP}^{-8} \mathrm{~mol} / \mathrm{L}$ group).

Sohlh 1 and Amh to evaluate the activation of primordial follicles. Four-day-old rat ovaries were treated with $10^{-7} \mathrm{~mol} / \mathrm{LV}$ VIP with or withoutVIP ${ }_{6-28}$ for 3 days, followed by RNA isolation and qRT-PCR. Results indicated that the mRNA expression of Soh/h 1 was significantly decreased in the VIP-treated group but increased with the addition of $\mathrm{VIP}_{6-28}$ (Fig. 5A). Meanwhile, the mRNA expression of $A m h$ increased in the VIP-treated group but decreased in the VIP +VIP ${ }_{6-28}$-treated group (Fig. 5B), indicating that VIP is indeed able to promote the development of primordial follicles into growing follicles.

\section{VIP activates primordial follicles through the ERK- mTOR signalling pathway}

To explore the possible mechanism by which VIP activates neonatal rat primordial follicles, ovaries were cultured for 3 days with VIP or VIP plus $10^{-8} \mathrm{~mol} / \mathrm{L}$ U0126 (VIP+U0126), which inhibits the phosphorylation of MEK or VIP plus $8.75 \times 10^{-8} \mathrm{~mol} / \mathrm{L}$ rapamycin
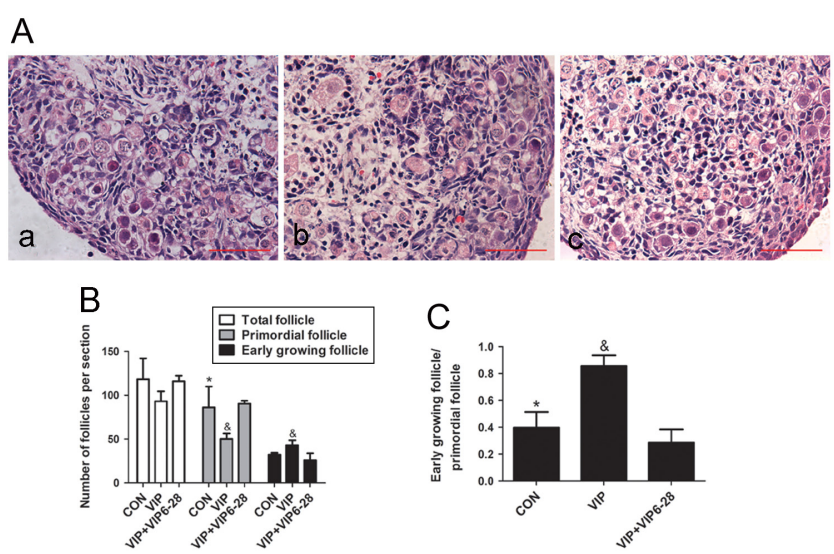

Figure $3 \mathrm{VIP}_{6-28}$ inhibits the activation of neonatal rat ovary by VIP. Four-day-old rat ovaries were cultured in basal medium without VIP (control group) or with $10^{-7} \mathrm{~mol} / \mathrm{L}$ VIP or VIP $+\mathrm{VIP}_{6-28}\left(5 \times 10^{-6} \mathrm{~mol} / \mathrm{L}\right)$ for 3 days. (A) The representative photos of all groups stained with hematoxylin and eosin: control (a), VIP (b), VIP+VIP ${ }_{6-28}$ (c). (B and C) The numbers of early-growing follicles and primordial follicles were counted, and their ratio was calculated. Scale bar: $50 \mu \mathrm{m}$. Values are mean \pm S.D. of at least three experiments. $\left({ }^{*} P<0.05\right.$ compared with the VIP-treated group, ${ }^{\&} P<0.05$ compared with the $\mathrm{VIP}+\mathrm{VIP}_{6-28}{ }^{-}$ treated group).

(VIP + Rapa), the inhibitor of mTORC1. As seen in Fig. 6, as indicated in $\mathrm{H} \& \mathrm{E}$ staining, treatment with either U0126 or rapamycin could decrease the ratio of GF/ $\mathrm{PF}$ as compared to that in the VIP-treated groups. The number of PCNA primordial-positive follicles and the expression of PCNA were also decreased as quantified by western blot (Fig. 7A, B and C). Meanwhile, TUNEL assay result showed that the occurrence of apoptosis increased with the presence of any of the two inhibitors (Fig. 7D and E).

The phosphorylation of ERK1/2 in the VIP groups significantly increased compared with that in the control group and the VIP + U0126 group but was similar to that in the VIP+Rapa groups. On the other hand, the phosphorylation of mTOR and its downstream protein, RPS6, were significantly increased compared to that in the other groups (Fig. 8B).

It is well known that primordial follicle initiation is accompanied by the activation of the phosphatidyinositol3-kinase (PI3K)/AKT/FOXO3Asignaling pathway in oocytes (Zhang \& Liu 2015). Hence, we examined the expression of AKT and FOXO3A phosphorylation. Result indicated that the phosphorylation of AKT and FOXO3A were significantly decreased in the VIP+U0126 and VIP + Rapa groups, reflecting less primordial follicle activation (Fig. 8A).

\section{Discussion}

Although much research has been done on the mechanism of follicular activation, the specific mechanism of primordial follicle initiation is still not 


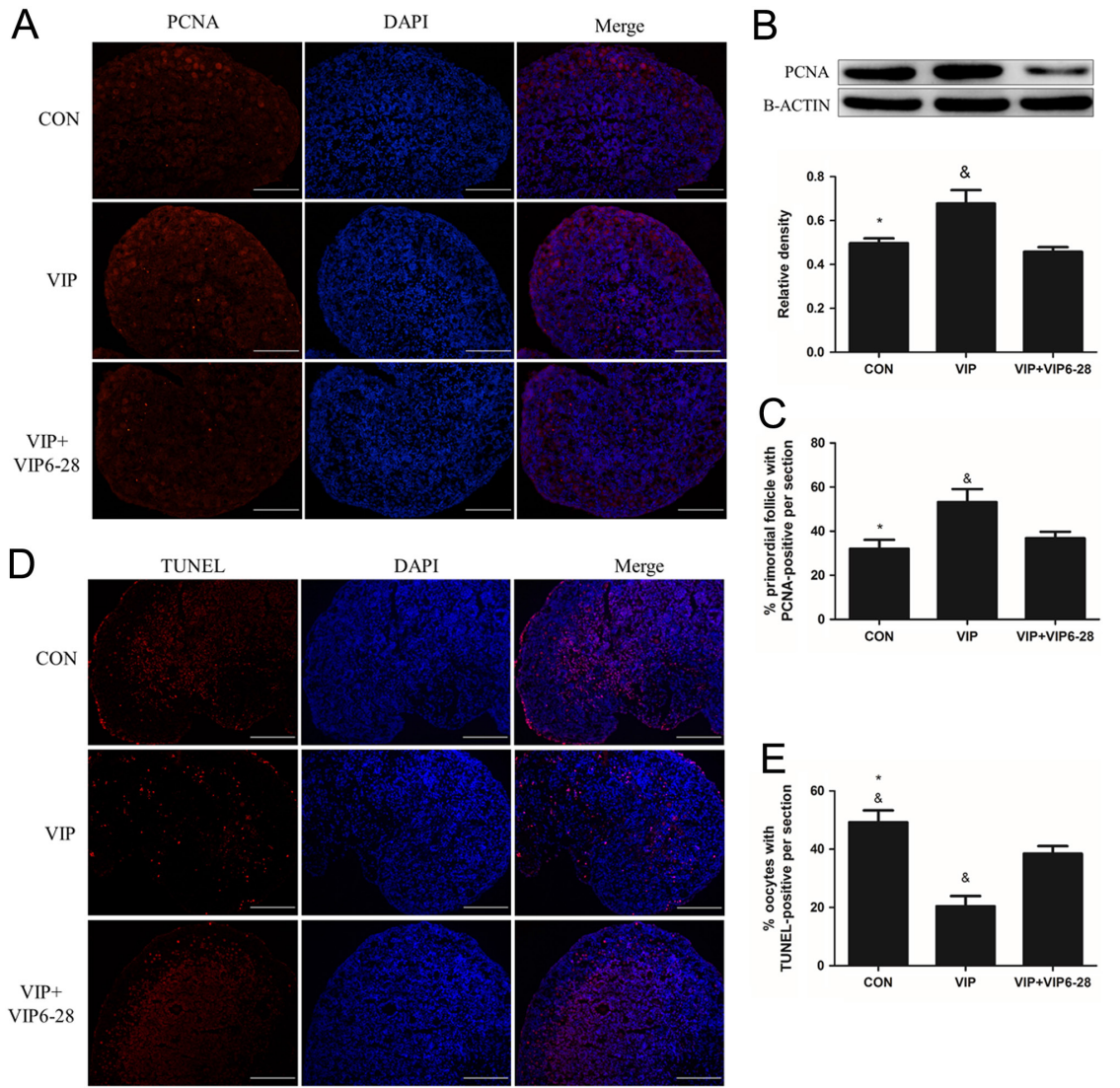

Figure 4 VIP promotes the proliferation of granular cells and inhibits the apoptosis of oocytes. Four-day-old ovaries were cultured with $10^{-7} \mathrm{~mol} / \mathrm{L}$ VIP or VIP + VIP ${ }_{6-28}$ $\left(5 \times 10^{-6} \mathrm{~mol} / \mathrm{L}\right)$ for 3 days. (A and D) PCNA staining and TUNEL assay were conducted in the three groups mentioned above for proliferation and apoptosis observation. (B) Western blot was conducted to quantify the expression of PCNA. (C and E) The percentage of primordial follicles or oocytes with PCNA-positive or TUNEL-positive section. Scale bar: $100 \mu \mathrm{m}$. Values are mean \pm S.D. of at least three experiments. $\left({ }^{*} P<0.05\right.$ compared with the VIP-treated group, ${ }^{\&} P<0.05$ compared with the VIP $+\mathrm{VIP}_{6-28}$-treated group). completely understood. Our study has demonstrated the ability of VIP in promoting primordial follicle activation through the ERK-mTOR signalling pathway in neonatal rat ovary, providing evidence and insight into the effect and mechanism of the microenvironment that is involved in the activation of primordial follicle.

Both VIP and its receptors had been found in preantral and antral follicles in the ovaries of several mammals. The expression of VIP is increased during luteinizing hormone surge in granular cells and follicular fluid. The mRNA transcripts for VIP receptors were identified in 22-day-old mice ovaries and in ovarian follicles at different developmental stages of pigs, as well as in ovarian extracts of human foetuses and women (Barberi et al. 2007, Gabbay-Benziv et al. 2012, Bukowski \& Wasowicz 2015). As for the proteins, VPAC1 was identified in follicles, mostly in the oocytes, of all human foetus samples (22 gestational weeks onward) and some samples from women and girls (Gabbay-Benziv et al. 2012). However, only a minority of the samples from all three sources contained follicular VPAC2 protein. Nonetheless, a number of other researches had stated that VPAC2 was in fact more widespread than VAPC1, with positive stain observed in nearly all tissues (Harmar et al. 2012). In our study, we demonstrated that the proteins for VIP receptors do exist in primordial follicles and in early-growing follicles of 4-day-old rat ovaries, both in the granular cells and oocytes, which is similar to the other mammals mentioned earlier. All these findings suggest that VIP may be involved in the growth and development of follicles. Although the staining of protein for VIP yielded negative result, we had since confirmed the presence of VIP mRNA in rat ovaries (data not shown). A previous study showed similar outcome whereby protein staining for the VIP system yielded weak result, especially in foetal samples (Gabbay-Benziv et al. 2012). We speculate that this may be due to the rapid degradation of the VIP peptide.

It was known that VIP could cause a dose-dependent increment in aromatase activity and in levels of cAMP prior to folliculogenesis (George \& Ojeda 1987), which
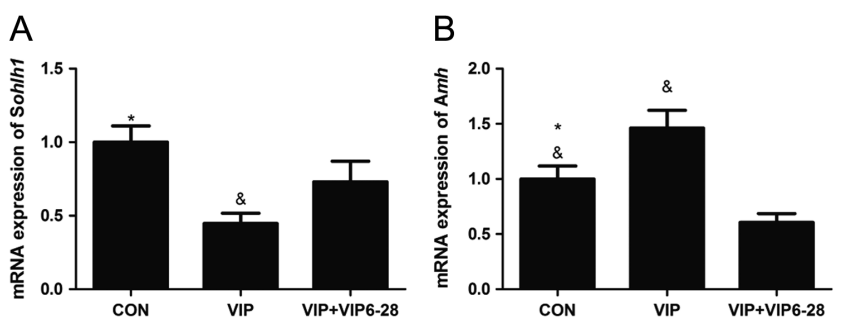

Figure 5 (A and B) The mRNA expression of Sohlh1 and Amh in neonatal ovary after treatment with $10^{-7} \mathrm{~mol} / \mathrm{LVIP}$ with or without $\mathrm{VIP}_{6-28}\left(5 \times 10^{-6} \mathrm{~mol} / \mathrm{L}\right)$ for 3 days. Values are mean \pm S.D. of at least three experiments. (Gene expression was normalized to Gapdh. $* P<0.05$ compared with the VIP group, \& $P<0.05$ compared with the $\mathrm{VIP}+\mathrm{VIP}_{6-28}$-treated group). 

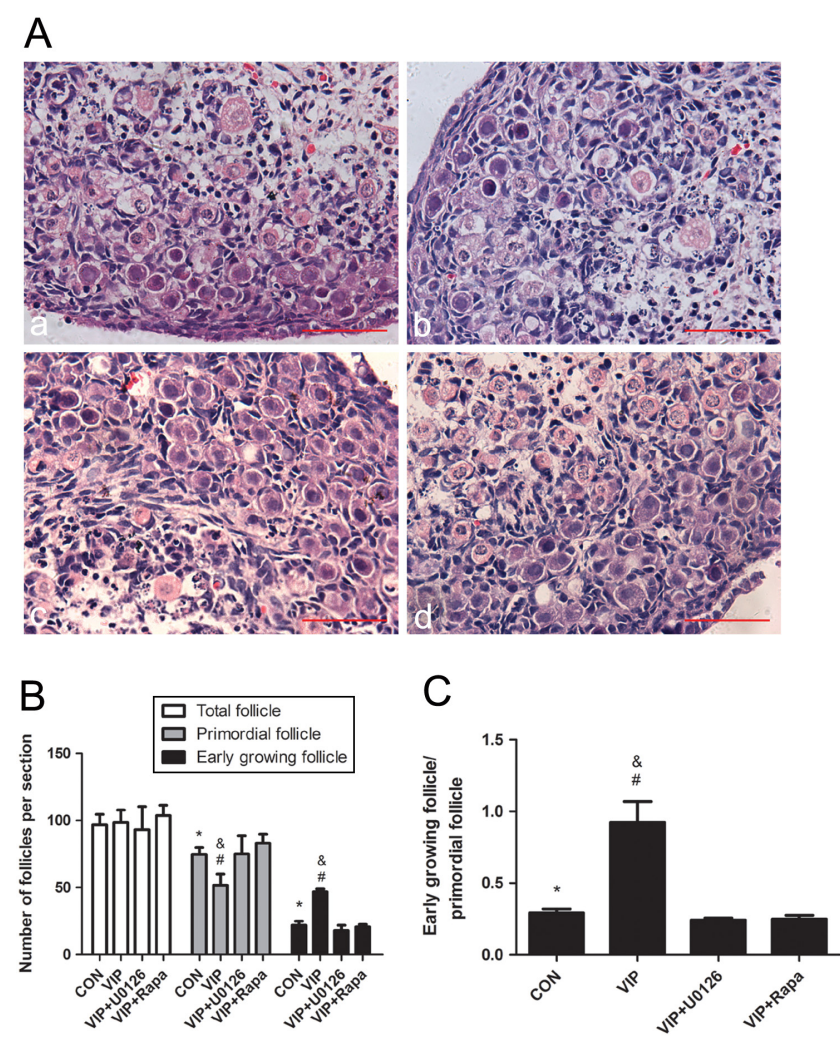

Figure 6 Effect of U0126 or rapamycin on VIP-induced follicle development. Four-day-old rat ovaries were cultured with (i) $10^{-7} \mathrm{~mol} / \mathrm{L}$ VIP, (ii)VIP plus $10^{-8} \mathrm{~mol} / \mathrm{L}$ U0126, or (iii) $\mathrm{VIP}+8.75 \times 10^{-8} \mathrm{~mol} / \mathrm{L}$ rapamycin for three days. (A) HE stained ovaries of different groups: control (a), VIP (b), VIP + U0126 (c), VIP + Rapa (d). (B and C) The numbers of early-growing follicles and primordial follicles were counted, and their ratio was calculated. Scale bar: $50 \mu \mathrm{m}$. Values are mean \pm S.D. of at least three experiments. $(* P<0.05$ compared with the VIP group, $\& P<0.05$ compared with the $\mathrm{VIP}+\mathrm{U} 0126$ group, ${ }^{\sharp} P<0.05$ compared with the VIP + Rapa group).

is related to the development of primordial follicles. This relationship suggests the probable role of VIP in promoting growth and development of early follicles. Mayerhofer et al. have reported that the mRNA transcript of FSH receptor was increased in 2-day-old rat ovaries that were cultured in vitro for $8 \mathrm{~h}$ with VIP. Follicular growth was then increased when rat ovaries were exposed to FSH (Mayerhofer et al. 1997). Since preantral follicles do not react with FSH, they would have possibly been promoted into antral follicles which are sensitive to FSH with the assistance of VIP. In addition, Bruno et al. also highlighted that VIP could improve the survival and development of caprine preantral follicles after in vitro tissue culture (Bruno et al. 2010), illustrating its possible crucial role in early follicle development and improving the quality of eggs that mature in vitro. Our study proves that VIP could promote the activation of primordial follicles in neonatal rat ovaries, induce granular cells proliferation and inhibit oocytes apoptosis. Furthermore, these effects were inhibited by $\mathrm{VIP}_{6-28}$, the antagonist of
VIP receptors, leading to the conclusion that VIP takes effect in neonatal ovaries via its specific receptors.

There are few reports about the mechanism by which VIP affects the development of follicles. We have demonstrated that VIP activates neonatal primordial follicle of rat through the ERK-mTOR signalling pathway. In vivo, VIP mainly acts through the CAMP-PKA pathway after binding to its specific receptors on cell membranes. Many proteins (including MAPK kinase) can be phosphorylated after PKA activation, resulting in a series of physiological effects, especially in the proliferation and differentiation of cells (Couvineau \& Laburthe 2012). It has been shown that VIP could mediate the proliferation of rat brain microvascular endothelial cells through the cAMP-PKA signalling pathway, supporting the claim mentioned earlier (Yang et al. 2013). Moreover, VIP increases the level of cAMP prior to folliculogenesis, while ERK and mTOR have been proven to be essential for primordial follicular activation. Therefore, our result is consistent with the findings of previous reports. Zhang et al. proved that the phosphorylation of mTOR in pregranular cells was the primary step for follicle recruitment in gene-knockout mice (Zhang et al. 2014). As VPAC exists in granular cell and oocytes, VIP may affect both during the activation of primordial follicles.

It is universally acknowledged that the PI3KPTEN (TENsin homology deleted in chromosome 10)-AKT-FOXO3 signalling pathway participates in the recruitment of primordial follicles in mammals, which governs the activation of oocyte in primordial follicles (Zhang \& Liu 2015). In this study, we have shown that the phosphorylation of AKT and FOXO3A was significantly increased. This reflects the possibility of oocyte activation followed by the activation of pre-granular cells or the direct effect of VIP on oocytes. However, some researches proposed that mTOR is located downstream of AKT in primordial follicle activation (Saxton \& Sabatini 2017), which is contradictory of the Western blot result in this study whereby the addition of rapamycin was able to decrease the phosphorylation of AKT. According to the study in gene-knockout mice by Zhang et al., it is possible that VIP first activates the mTOR signalling pathway in pre-granular cells, which subsequently leads to the activation of PI3K/AKT signalling pathway in oocyte (Zhang et al. 2014).

Some researchers had attempted to use stimulators of AKT or PI3K combined with inhibitor of PTEN to activate dormant follicles in the treatment of patients with primary ovarian insufficiency as part of the IVA strategy (Suzuki et al. 2015, Zhai et al. 2016). Although follicle growth appeared in some patients, successful pregnancies were rare, only two in 37 patients or one in 14 patients. The PTEN inhibition could result in increased DNA damage and impaired DNA repair capacity in ovarian follicles while promoting bovine non-growing follicle activation (Maidarti et al. 2019). We do acknowledge that the 
A

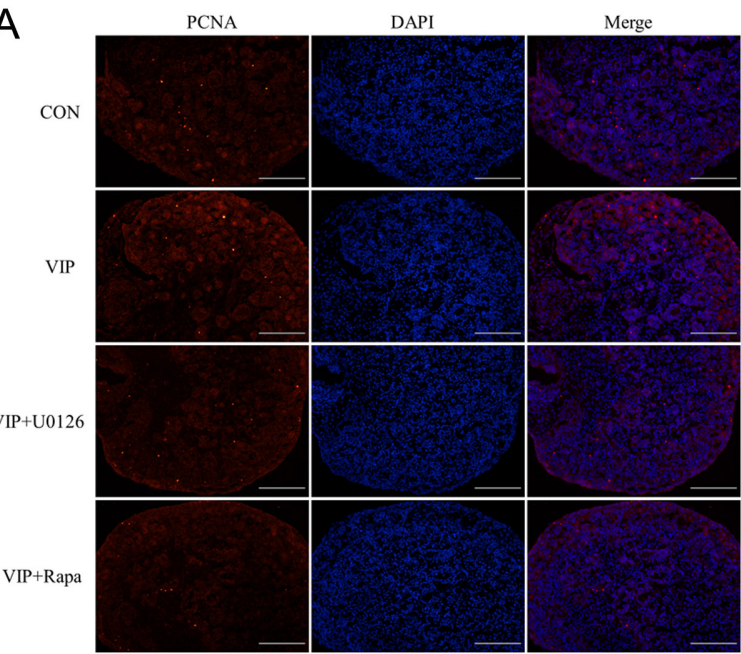

D

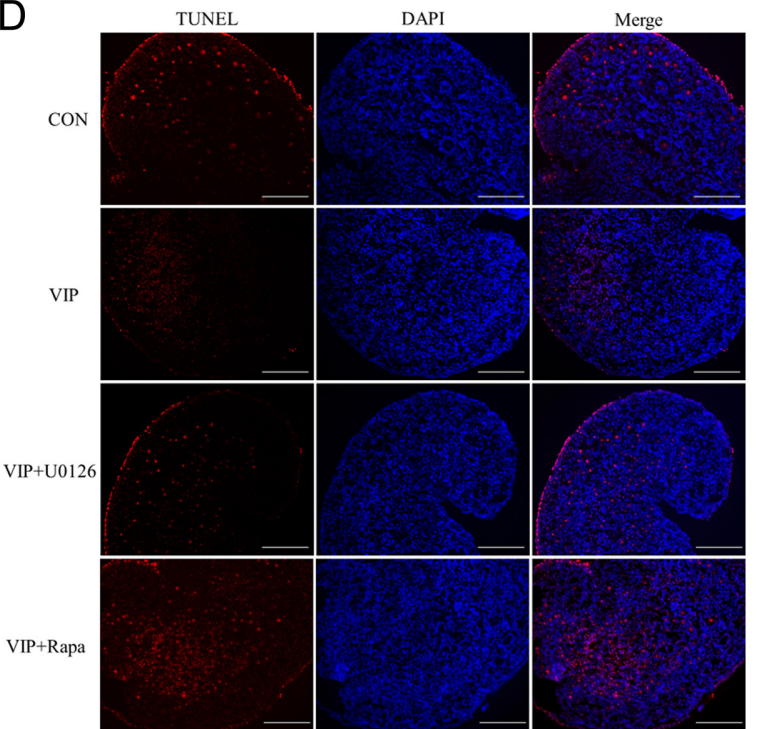

B

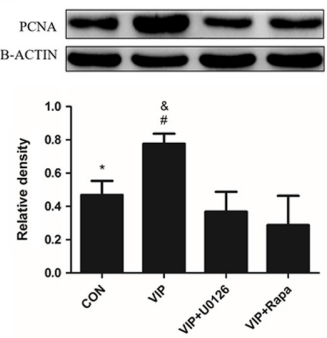

C
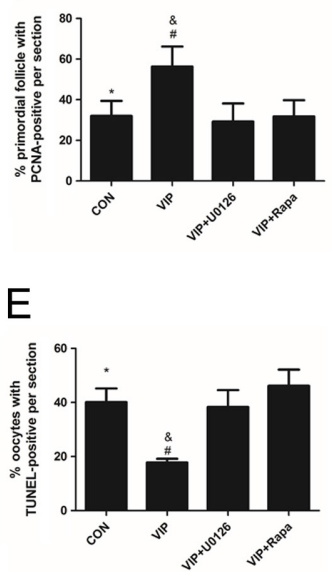

Figure 7 U0126 or rapamycin inhibits the VIP-induced proliferation of granular cells and promotes oocyte apoptosis. Ovaries were cultured with (i) $10^{-7} \mathrm{~mol} / \mathrm{L} \mathrm{VIP,} \mathrm{(ii)}$ $\mathrm{VIP}+10^{-8} \mathrm{~mol} / \mathrm{L}$ U0126 or (iii) VIP plus $8.75 \times 10^{-8} \mathrm{~mol} / \mathrm{L}$ rapamycin for 3 days. (A and D) PCNA staining and TUNEL assay were conducted for proliferation and apoptosis observation. (B) Western blot was conducted to quantify the expression of PCNA. (C and E) The numbers of primordial follicles with positive PCNA staining and oocytes with positive TUNEL staining were counted. Scale bar: $100 \mu \mathrm{m}$. Values are mean \pm S.D. of at least three experiments. $\left({ }^{*} P<0.05\right.$ compared with the VIP group, ${ }^{\&} P<0.05$ compared with the VIP + U0126 group, ${ }^{\sharp} P<0.05$ compared with the VIP+ Rapa group). activation of primordial follicles is indeed complex. The PI3K-PTEN-AKT-FOXO3 or ERK-mTOR or other signalling pathways may only be a small component of the full mechanism which is yet to be fully elucidated. Thus, it is difficult to acquire full success by relying only on stimulating those signalling pathways. There are proposals that the microenvironment and the local factors surrounding individual primordial follicle are likely to play defining roles in deciding the fates of these follicles as well, since many growth factors have been reported to be functional in regulating the activation of primordial follicles in vitro (Zhang \& Liu 2015). Therefore, the success rate of IVA might be improved through the right combination of useful growth factors and stimulators. Moreover, considering the risk of ovarian tissue auto-replantation in cancer patients, it will be beneficial if mature oocytes could be acquired in vitro. It is necessary to study the microenvironment as IVA is of great importance as the first step to treating infertility. VIP may emerge as one of the growth factors that could be used in IVA to obtain matured oocytes in vitro.

Last but not least, the in vitro culture system in our study may not be sufficient for healthy follicle development, since quite a few early-growing follicles appeared to be disordered in terms of granulosa cell arrangement, especially in VIP-treated ovaries. It might be due to lack of necessary factors, like leukaemia inhibitory factor, basic fibroblast growth factor and platelet-derived growth factor, which had been reported to promote follicle development (Zhang \& Liu 2015). Besides, there were more early-growing follicles that were activated by VIP than primordial follicles. Therefore, it is reasonable for a larger number of healthy oocytes to be observed in the control group as compared with that in VIP-treated group. Other necessary factors must be taken into account for the application of VIP in IVA to stimulate and retain healthy follicles. 

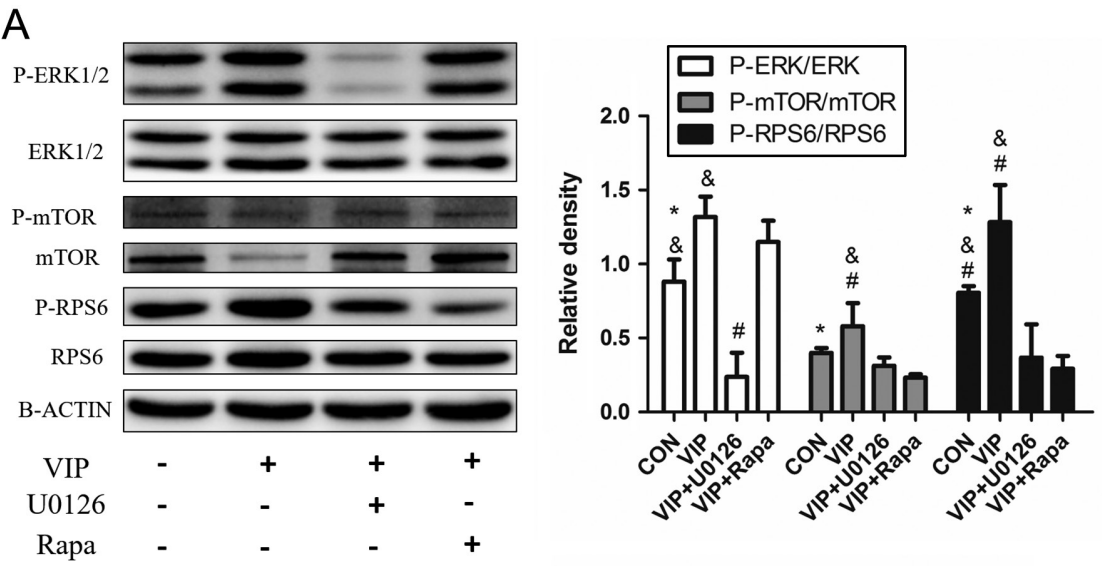

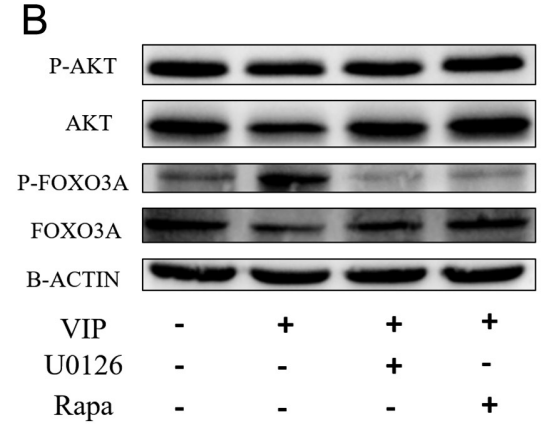

\section{Conclusion}

This study demonstrated the ability of VIP to activate primordial follicles of neonatal rat ovary in vitro, likely through the ERK-mTOR signalling pathway. Our findings provide a new strategy for in vitro activation of dormant follicles.

\section{Supplementary data}

This is linked to the online version of the paper at https://doi.org/10.1530/REP-18-0466.

\section{Declaration of interest}

The authors declare that there is no conflict of interest that could be perceived as prejudicing the impartiality of the research reported.

\section{Funding}

This work was supported by grants from Sun Yat-Sen University Clinical Research 5010 Program (2016004), from Sun Yat-Sen Clinical Research Cultivating Program, Guangdong Science and Technology Department (2017B030314026).

\section{Author contribution statement}

Song Li, Qi Fan and Qingxue Zhang contributed significantly to the conception and design of this work. Haiyan Lin and Qi Qiu contributed to data analyses. Song Li, Yanqiu Xie and Yihua Liang wrote the manuscript. All authors revised the paper and approved the final version.

\section{Acknowledgement}

The authors would like to thank the State Key Laboratory of Ophthalmology, Zhongshan Ophthalmic Centre of Sun Yat-sen University for providing the instruments for research.

\section{References}

Barberi M, Muciaccia B, Morelli MB, Stefanini M, Cecconi S \& Canipari R 2007 Expression localization and functional activity of pituitary adenylate cyclase-activating polypeptide, vasoactive intestinal polypeptide and their receptors in mouse ovary. Reproduction 134 281-292. (https://doi. org/10.1530/REP-07-0051)

Bruno JB, Celestino JJ, Lima-Verde IB, Matos MH, Lima LF, Name KP, Araújo VR, Saraiva MV, Martins FS, Campello CC et al. 2010 Vasoactive intestinal peptide improves the survival and development of caprine preantral follicles after in vitro tissue culture. Cells Tissues Organs 191 414-421. (https://doi.org/10.1159/000272317)

Bukowski R \& Wąsowicz K 2015 Expression of VPAC1 receptor at the level of $\mathrm{mRNA}$ and protein in the porcine female reproductive system. Polish Journal of Veterinary Sciences 18 199-206. (https://doi.org/10.1515/ pjvs-2015-0025) 
Cecconi S, Rossi G, Barberi M, Scaldaferri L \& Canipari R 2004 Effect of pituitary adenylate cyclase-activating polypeptide and vasoactive intestinal polypeptide on mouse preantral follicle development in vitro. Endocrinology 145 2071-2079. (https://doi.org/10.1210/en.2003-1004)

Chen N, Li Y, Wang W, Ma Y, Yang D \& Zhang Q 2013 Vasoactive intestinal peptide can promote the development of neonatal rat primordial follicles during in vitro culture. Biology of Reproduction 88 12. (https:// doi.org/10.1095/biolreprod.111.098335)

Couvineau A \& Laburthe Mb 2012 VPAC receptors: structure, molecular pharmacology and interaction with accessory proteins. British Journal of Pharmacology 166 42-50. (https://doi.org/10.1111/j.14765381.2011.01676.x)

Davis RJ 2000 Signal transduction by the JNK group of MAP kinases. Cell 103 239-252. (https://doi.org/10.1016/S0092-8674(00)00116-1)

Dayal M, Sagar S, Chaurasia A \& Signgh U 2014 Anti-mullerian hormone: a new marker of ovarian function. Journal of Obstetrics and Gynaecology of India 64 130-133. (https://doi.org/10.1007/s13224-013-0482-3)

Gabbay-Benziv R, Ao A, Fisch B, Zhang L, Oron G, Kessler-Icekson G, Ben-Haroush A, Krissi H \& Abir R 2012 Vasoactive intestinal peptide and its receptors in human ovarian cortical follicles. PLOS ONE 7 e37015. (https://doi.org/10.1371/journal.pone.0037015)

George FW \& Ojeda SR 1987 Vasoactive intestinal peptide enhances aromatase activity in the neonatal rat ovary before development of primary follicles or responsiveness to follicle-stimulating hormone. PNAS 84 5803-5807. (https://doi.org/10.1073/pnas.84.16.5803)

Harmar AJ, Fahrenkrug J, Gozes I, Laburthe M, May V, Pisegna JR, Vaudry D, Vaudry H, Waschek JA \& Said SI 2012 Pharmacology and functions of receptors for vasoactive intestinal peptide and pituitary adenylate cyclase-activating polypeptide: IUPHAR review 1. British Journal of Pharmacology 166 4-17. (https://doi.org/10.1111/j.14765381.2012.01871.x)

Hulshof SC, Dijkstra G, Van der Beek EM, Bevers MM, Figueiredo JR, Beckers JF \& Van den Hurk R 1994 Immunocytochemical localization of vasoactive intestinal peptide and neuropeptide $\mathrm{Y}$ in the bovine ovary. Biology of Reproduction 50 553-560. (https://doi.org/10.1095/ biolreprod50.3.553)

Jagarlamudi K \& Rajkovic A 2012 Oogenesis: transcriptional regulators and mouse models. Molecular and Cellular Endocrinology 356 31-39. (https://doi.org/10.1016/j.mce.2011.07.049)

Kim S, Lee Y, Lee S \& Kim T 2018 Ovarian tissue cryopreservation and transplantation in patients with cancer. Obstetrics and Gynecology Science 61 431-442. (https://doi.org/10.5468/ogs.2018.61.4.431)

Ladanyi C, Mor A, Christianson MS, Dhillon N \& Segars JH 2017 Recent advances in the field of ovarian tissue cryopreservation and opportunities for research. Journal of Assisted Reproduction and Genetics 34 709-722. (https://doi.org/10.1007/s10815-017-0899-1)

Maidarti M, Clarkson YL, McLaughlin M, Anderson RA \& Telfer EE 2019 Inhibition of PTEN activates bovine non-growing follicles in vitro but increases DNA damage and reduces DNA repair response. Human Reproduction 34 297-307. (https://doi.org/10.1093/humrep/dey354)

Mayerhofer A, Dissen GA, Costa ME \& Ojeda SR 1997 A role for neurotransmitters in early follicular development: induction of functional follicle-stimulating hormone receptors in newly formed follicles of the rat ovary. Endocrinology 138 3320-3329. (https://doi.org/10.1210/ endo.138.8.5335)
Onoue S, Misaka S \& Yamada S 2008 Structure-activity relationship of vasoactive intestinal peptide (VIP): potent agonists and potential clinical applications. Naunyn-Schmiedebergs' Archives Pharmacololy 377 579-590. (https://doi.org/10.1007/s00210-007-0232-0)

Pangas SA, Choi Y, Ballow DJ, Zhao Y, Westphal H, Matzuk MM \& Rajkovic AY 2006 Oogenesis requires germ cell-specific transcriptional regulators Sohlh1 and Lhx8. PNAS 103 8090-8095. (https://doi. org/10.1073/pnas.0601083103)

Pearson G, Robinson F, Beers Gibson T, Xu BE, Karandikar M, Berman K \& Cobb MH 2001 Mitogen-activated protein (MAP) kinase pathways: regulation and physiological functions. Endocrine Reviews 22 153-183. (https://doi.org/10.1210/edrv.22.2.0428)

Saxton RA \& Sabatini DM 2017 mTOR signaling in growth, metabolism, and disease. Cell 168 960-976. (https://doi.org/10.1016/j.cell.2017.02.004)

Streiter S, Fisch B, Sabbah B, Ao A \& Abir R 2016 The importance of neuronal growth factors in the ovary. Molecular Human Reproduction 22 3-17. (https://doi.org/10.1093/molehr/gav057)

Suzuki N, Yoshioka N, Takae S, Sugishita Y, Tamura M, Hashimoto S, Morimoto Y \& Kawamura K 2015 Successful fertility preservation following ovarian tissue vitrification in patients with primary ovarian insufficiency. Human Reproduction 30 608-615. (https://doi. org/10.1093/humrep/deu353)

Yang J, Shi QD, Song TB, Feng GF, Zang WJ, Zong CH \& Chang L 2013 Vasoactive intestinal peptide increases VEGF expression to promote proliferation of brain vascular endothelial cells via the CAMP/PKA pathway after ischemic insult in vitro. Peptides 42 105-111. (https://doi. org/10.1016/j.peptides.2013.01.007)

Zhai J, Yao G, Dong F, Bu Z, Cheng Y, Sato Y, Hu L, Zhang Y, Wang J, Dai S et al. 2016 In vitro activation of follicles and fresh tissue autotransplantation in primary ovarian insufficiency patients. Journal of Clinical Endocrinology and Metabolism 101 4405-4412. (https://doi. org/10.1210/jc.2016-1589)

Zhang H \& Liu K 2015 Cellular and molecular regulation of the activation of mammalian primordial follicles: somatic cells initiate follicle activation in adulthood. Human Reproduction Update21 779-786. (https://doi. org/10.1093/humupd/dmv037)

Zhang H, Risal S, Gorre N, Busayavalasa K, Li X, Shen Y, Bosbach B, Brännström M \& Liu K 2014 Somatic cells initiate primordial follicle activation and govern the development of dormant oocytes in mice. Current Biology 24 2501-2508. (https://doi.org/10.1016/j. cub.2014.09.023)

Zhao Y, Zhang Y, Li J, Zheng N, Xu X, Yang J, Xia G \& Zhang M 2018 MAPK3/1 participates in the activation of primordial follicles through mTORC1-KITL signaling. Journal of Cellular Physiology 233 226-237. (https://doi.org/10.1002/jcp.25868)

Received 2 September 2018

First decision 10 October 2018

Revised manuscript received 20 February 2019

Accepted 26 February 2019 